\title{
LA TRASCENDENCIA DE LAS POLÍTICAS EDUCATIVAS: UNA MEDIACIÓN PARA EL DESARROLLO HUMANO.
}

\author{
LORENA OSIRIS SUAZO ALVARENGA ${ }^{1}$ \\ MARLENNE YAMILETH ORDÓÑEZ ${ }^{2}$ \\ ROSA ELIA SABILLÓN ${ }^{3}$ \\ ORLANDO MARTÍNEZ ARRAZOLA ${ }^{4}$ \\ YURI FORTÍN ${ }^{5}$
}

Doctorandos en Ciencias del Desarrollo Humano, Universidad Nacional Autónoma de Honduras

DOI: $10.5377 /$ rpdd.v6i1.12444

Recibido: agosto, 2021

Aceptado: septiembre, 2021

\section{RESUMEN}

$\mathrm{L}$ a educación como mediación para el desarrollo, que se incluye en estas iniciativas, es un componente clave en la política social del Estado. A partir del reconocimiento de la educación como un derecho fundamental de todo ciudadano es que se originan sus obligaciones en el diseño e implementación de acciones gubernamentales que buscan dar respuestas a las demandas de la sociedad. Estos esfuerzos se evidencian en las políticas públicas que los países generan para abordar los diversos problemas que enfrentan. Entender el rediseño de las políticas educativas y la generación de impacto en la reconfiguración del sistema educativo no es posible sin tener en cuenta a los actores o agentes educativos. El presente artículo tiene como propósito generar una reflexión en torno a la trascendencia de las políticas educativas como una dimensión del desarrollo humano. Se resalta la participación ciudadana como elemento esencial que contribuirá holísticamente a la resignificación del sistema educativo, el cual está supeditado en buena parte por la buena gobernanza, rediseño de las políticas educativas y por un cuerpo de actores estratégicos y democráticos.

1MSc. LORENA OSIRIS SUAZO ALVARENGA, Docente Universidad Nacional Autónoma de Honduras, lorena.suazo@unah.edu.hn, Orcid: https://orcid.org/0000-0003-4712-6420.

2MSC. MARLENNE YAMILETH ORDÓÑEZ, Docente Universidad Nacional Autónoma de Honduras, marlene. ordonez@unah.edu.hn, Orcid: https://orcid.org/0000-0001-7395-6968.

3MSc. ROSA ELIA SABILLÓN, Docente Universidad Nacional Autónoma de Honduras, rosa.sabillon@unah. edu.hn, Orcid: https://orcid.org/0000-0002-9977-6671.

4MSc. ORLANDO MARTÍNEZ ARRAZOLA, Docente Universidad Metropolitana de Honduras, ojmartinez@ unah.edu.hn, Orcid: https://orcid.org/0000-0002-9848-9259.

5MSc. YURI FORTÍN, Docente Universidad Metropolitana de Honduras, yufortindoc2018@gmail.com, Orcid: https://orcid.org/0000-0001-8175-2383. 
Las políticas públicas para la sostenibilidad de los Sistemas Nacionales de Educación requieren llevar como propósitos fundamentales el uso eficiente y transparente de los recursos materiales e inmateriales asignados, la construcción de una educación inclusiva de la diversidad, el acceso de la mayoría o a los sectores que requieran de una educación formal o educación no formal, técnica no universitaria, educación técnica universitaria, también universitaria en licenciaturas, especialidades, maestrías, doctorados y posdoctorados.

\section{PALABRAS CLAVE}

Política pública educativa, participación ciudadana, sistema educativo, desarrollo humano, ética.

\section{INTRODUCCIÓN}

\section{Contextualización de la educación en el marco de las políticas públicas}

El mundo enfrenta una era planetaria que se desarrolla en medio de una serie de desafíos y tensiones y donde la ciencia, la técnica, la industria y el interés económico se enfrentan a las ideas humanistas y libertadoras que la sociedad está exigiendo. Ante esta realidad es necesario plantear el surgimiento de una sociedad mundial que promueva la gobernabilidad para un mundo mejor, para lo cual recurre a la educación como fundamento del proceso de cambio hacia una mayor humanización del ser humano.

Gran parte de los esfuerzos globales para contribuir al desarrollo de las economías de ingresos bajo y medio están orientados a proveer los recursos y la información que necesitan en su camino por la vida, para que las personas puedan dirigir mejor su propio destino. Las iniciativas promovidas por los organismos internacionales (ONU, PNUD, FMI, BID) como la Estrategia de Reducción de la Pobreza (ERP), los Objetivos del Desarrollo del Milenio (ODM-2015) y ahora los Objetivos de Desarrollo Sostenible (ODS-2030) son parte de estos modelos que han tenido como fin primordial promover un mejoramiento de la calidad de vida de las personas (UNESCO, 2020).

La educación como elemento mediador que se incluye en estas iniciativas es un componente clave en la política social del Estado. Es un hecho muy complejo, por el conjunto y variedad de factores, actores y funciones que la integran; y delicado 


\section{LA TRASCENDENCIA DE LAS POLÍTICAS EDUCATIVAS: UNA MEDIACIÓN PARA EL DESARROLLO HUMANO.}

por los vigorosos impulsos que lo transitan y por la diversidad de repercusiones y consecuencias que sus acciones y efectos crean en la sociedad.

A partir del reconocimiento de la educación como un derecho fundamental de todo ciudadano se originan las obligaciones del Estado en el diseño e implementación de políticas y acciones que buscan dar respuestas a las demandas de la sociedad. Estos esfuerzos se evidencian en las políticas públicas que los países generan para abordar los diversos problemas que enfrentan. Las políticas públicas educativas, particularmente, tienen como propósito garantizar la educación como derecho humano que tienen todas las personas por el simple hecho de nacer.

Esta aspiración de la educación como un instrumento de mejora en la calidad de vida de las personas se evidencia en los enfoques de desarrollo (crecimiento económico, crecimiento con redistribución, satisfacción de las necesidades, ajuste estructural, neoliberalismo, expansión de capacidades, desarrollo de lo humano y desarrollo sustentable) donde se concibe que este concepto posibilita un autodesarrollo de todas las comunidades nacionales, lo que implica una interdependencia entre naciones. Ahora bien, si se analizan los esfuerzos gubernamentales en relación con políticas educativas en el marco de los enfoques de desarrollo, se aprecia que la educación básica resulta ser la mayor necesidad que comparten mayoritariamente los países emergentes y a los que estos enfoques se han propuesto como meta mejorar (Lorente Rodríguez, 2019).

Consecuentemente, con la elección de priorizar la educación básica como estrategia de primer orden y herramienta para erradicar o, al menos, minimizar las cifras de analfabetismo, así como los elevados índices de repetición, rezago y abandono educativos son las estrategias de calidad y equidad las que ocupan lugares prioritarios en las agendas educativas latinoamericanas.

Desde el inicio de la década de los 90, en el siglo XX, varios países de Latinoamérica emprendieron sus modelos, políticas educativas y estrategias de acción hacia una mejora de la calidad educativa bajo las recomendaciones de la Organización de las Naciones Unidas para la Educación, la Ciencia y la Cultura (UNESCO) establecidas en la Declaración Mundial sobre Educación para Todos y el Marco de Acción para Satisfacer las Necesidades Básicas de Aprendizaje (UNESCO, 2020, pp. 12-13). 
De acuerdo con la Comisión Económica para América Latina y el Caribe (CEPAL) la región pasa por afrontar los desafíos que anticipa el nuevo siglo relacionados, principalmente, con la consecución de una sociedad del conocimiento y el progreso tecnológico sin descuidar los desafíos todavía pendientes del siglo $\mathrm{XX}$, tales como cobertura, acceso, progresión y conclusión de los distintos niveles educativos (Lorente Rodríguez, 2019). Lo anterior puede verse reflejado en buena parte en los documentos legislativos de las diferentes reformas educativas acometidas, así como en los discursos de gobiernos, cumbres de ministros de educación y organismos internacionales.

Si se valoran las propuestas de estos organismos internacionales en torno a acciones de mejora en la dimensión de la educación se identifica su presencia con intereses más económicos (Bancos de Desarrollo, OCDE, Banco Mundial y Fondo Monetario Internacional) lo que se contrasta con aquellos cuyas políticas se focalizan en torno a los aspectos más sociales (CEPAL, PNUD, OIT), conformando dos flancos con aspiraciones divergentes. Por su parte, las ONGs se erigen como instrumentos privilegiados para abordar los proyectos de índole más político y cultural de educación, a la vez que promueven ideas renovadoras y alertan a la comunidad internacional sobre los nuevos desafíos educativos que afronta América Latina, en tanto que la UNESCO aborda por su parte, los programas de enseñanza que evidencien sinergias con la variable más humana, cultural y sostenible (UNESCO, 2020). Las variables sociales, por tanto, están presentes, demostrando con ello la necesaria implementación del elemento educativo y social.

El escenario desafiante que en la actualidad se vive en términos educativos en la región latinoamericana se evidencia en las cifras que la UNESCO ofrece. De acuerdo con este organismo alrededor del $63 \%$ de las personas jóvenes finalizan la escuela secundaria, pero en 20 países latinoamericanos las probabilidades de que lo hagan los alumnos del $20 \%$ más rico, son cinco veces más altas que las del $20 \%$ más pobre. Las tasas de asistencia a la escuela son más bajas para las personas jóvenes con discapacidad, los hablantes de lenguas indígenas y las personas afrodescendientes. La mitad de los alumnos de 15 años en América Latina no alcanza el nivel mínimo de competencia en lectura, del 55\% de los países de América Latina y el Caribe tienen planes o estrategias sectoriales de educación que se refieren a la educación inclusiva como una prioridad (UNESCO, 2020).

Por su parte, el esfuerzo que un gobierno realiza por mejorar las condiciones de vida de sus habitantes se puede apreciar en los fondos estatales destinados a la 


\section{LA TRASCENDENCIA DE LAS POLÍTICAS EDUCATIVAS: UNA MEDIACIÓN PARA EL DESARROLLO HUMANO.}

educación. Según la UNESCO (2020) hasta el año 2019 se logró apreciar que tres países de la región latinoamericana (Belice, Costa Rica y Monserrat) figuran entre los 10 países más destacados del mundo dedicando como mínimo el 7\% de su PIB a la educación. Sin embargo, el gasto en educación medido como porcentaje del PIB en América Latina y el Caribe, aumentó del 3.9\% en 2000 a 5.6\% en 2017, todavía persiste una disparidad en los presupuestos de los países en vías de desarrollo donde la mayoría del gasto público se destina al pago de salarios de los burócratas.

Se aprecia que el énfasis en la educación básica y los bajos niveles de financiamiento de la educación son componentes que se suman a los débiles sistemas educativos que existen y que todavía permanecen ausentes en las iniciativas gubernamentales, provocando un incremento en la brecha que separa a las naciones desarrolladas y a las que están en vías de desarrollo en el marco del proceso de adaptación a las transformaciones que la globalización exige en la actualidad. Es en este sentido que Lorente Rodríguez (2019) sostiene que esta realidad es en gran parte fruto de la desigualdad perenne que caracteriza a la región, cuyas brechas sociales, económicas y propiamente educativas cada vez son más amplias y tienden a retroalimentarse mutuamente. Los retos educativos que enfrenta la región latinoamericana son cada vez mayores, los sistemas educativos evidencian una serie de deficiencias, configurando distintas agrupaciones de naciones que caminan a distintos ritmos hacia el propósito de universalizar el conocimiento.

Honduras forma parte de este conjunto de países donde las reformas en el sector educativo han carecido de un apoyo decidido del Estado a través de los gobiernos $\mathrm{y}$, sobre todo, no se ha logrado que todos los actores validen, legitimen y acepten las reformas educativas. Los cambios de gobierno provocan la creación de nuevas agendas nacionales, esto posiblemente no le permite al país articular un proyecto con otro (Cáceres Valladares, 2018). El escenario actual del sistema educativo hondureño evidencia los rezagos sobre el compromiso del Estado de Honduras en brindar el acceso a una educación de buena calidad que permita a las nuevas generaciones desarrollar sus potencialidades e incorporarse en el sistema productivo con las competencias que demanda el mercado mundial (Rojas, 2020, p. 12).

Las políticas públicas requieren considerar el vector social (atención más específica para las poblaciones indígenas y rurales, programas que combatan problemas consustanciales como el rezago, repetición y ausentismo estudiantil, políticas educativas focalizadas sobre la situación de la mujer y su papel en la sociedad latinoamericana) y también político (educación popular y educación liberadora- 
emancipadora) a la vez que no renuncie a su cultura y tradiciones y que incluyan una política educativa verde para el desarrollo económico que proporcionará mejor calidad de vida, y esta calidad estará en armonía con el entorno.

\section{Resignificación del sistema educativo y el rol de los actores}

En general, una propuesta reformista de política educativa en la región se confrontará con un contexto educativo a nivel local y regional con grandes desigualdades sociales. El informe La crisis de la desigualdad, América Latina y el Caribe en la encrucijada (Banco Interamericano de Desarrollo, 2020) revela entre sus principales hallazgos que las desigualdades educativas están profundamente determinadas por el ingreso económico de los padres de los estudiantes. Según el mismo estudio en el país promedio de América Latina y el Caribe, el 10\% más rico de la población gana 22 veces más que el $10 \%$ más pobre, mientras que el coeficiente de Gini promedio se revela de 0,46 . Lo que muestra cierta heterogeneidad en la región: Brasil, Honduras y Panamá se encuentran entre los más desiguales, mientras que el Salvador, Uruguay y Argentina están entre los más igualitarios.

Ante la desigualdad social y educativa según el estudio antes citado, se presentan los siguientes desafíos de acuerdo a lo planteado por Vargas (2019) las opciones de política que se diseñen para la educación en la actual coyuntura están llamadas a reconocer deudas históricas con los grupos más postergados para garantizar su derecho a la educación, tanto en lo referido a la disponibilidad y el acceso a oportunidades de aprendizaje, como a la provisión de una educación de calidad, relevante y adaptada a sus condiciones, necesidades y aspiraciones.

En armonía con el autor anterior, el sociólogo Bauman (2011) advierte que:

...el compuesto explosivo que forman la desigualdad social en aumento y el creciente sufrimiento humano relegado al estatus de colateralidad (puesto que la marginalidad, la externalidad y la cualidad descartable no se han introducido como parte legítima de la agenda política) tiene todas las calificaciones para ser el más desastroso entre los incontables problemas potenciales que la humanidad puede verse obligada a enfrentar, contener y resolver durante el siglo en curso. (p.18)

Entender el rediseño de las políticas educativas y la generación de su impacto en la reconfiguración del sistema educativo no es posible sin tener en cuenta a los actores o agentes educativos partiendo de la premisa conforme a F. Reimers (2000) quien expresa que: 


\section{LA TRASCENDENCIA DE LAS POLÍTICAS EDUCATIVAS: UNA MEDIACIÓN PARA EL DESARROLLO HUMANO.}

...la integración de nuevos actores sociales en la definición de políticas educativas para el nuevo milenio asegura la construcción solidaria de comunidades, libres y pacíficas, bajo el precepto de que la igualdad de oportunidades educativas dará legitimidad a la democracia en la región. (p.11)

El Estado como principal entidad política se sigue considerando un actor clave que brinda legitimidad en los niveles de concreción de la política educativa y en la reconfiguración del sistema educativo. En ese sentido, el autor Jellnek, citado por Bechara Llanos y Abraham Zamir (2015), propone:

...una configuración desde una asimilación objetiva del sentido estatal, esta no puede estar alejada de la realidad del mundo por fuera de las subjetividades individuales o del mismo hombre en sentido particular, infiere, que, si bien es importante la estructura objetiva del Estado, esta no puede ser exclusiva a la lectura de la ciencia estatal, ya que la plenitud del mundo subjetivo del hombre frente a la visión del Estado se traslada hacia un conocimiento en sentido objetivo-subjetivo. (p.72)

Las consideraciones, conforme a lo que corresponde al rol del Estado, se deben entender bajo el marco de una innovación de la gestión democrática en esta materia (repensando la participación ciudadana), ya que es vital el tránsito desde una gobernanza convencional hasta la procura de eliminar privilegios, aumentando con mayor énfasis la legitimidad de las acciones políticas, logrando liderazgos corresponsables, empoderando tecnológicamente a los actores claves y fortaleciendo a todos los gestores educativos que impulsan, implementan y evalúan la política pública.

Cabe resaltar, que los modelos de participación más viables para este tipo de gobernanza no convencional son los modelos mixtos: participativo con criterios deliberativos. Según Dacombe y Pharvin (2021) este tipo de modelo podría representar el futuro "...por su capacidad para permitir un mayor grado de control popular sobre las acciones de los funcionarios y representantes públicos de lo que sería factible bajo una comprensión más limitada de la democracia" (p. 4). Además, proporciona una capa adicional de escrutinio sobre las acciones de los funcionarios públicos al aumentar el potencial para la participación y para que se puedan escuchar las voces disidentes y que las figuras públicas den cuenta de sus acciones. Del mismo modo, cualquier comportamiento errado podría quedar expuesto de la misma manera. 
Este enfoque tiene solidez dadas las diferentes formas de participación permitidas, porque es parte de la diversidad y la perspectiva para la acción política, conforme al autor J. Subirats (2011) quien hace referencia a que:

Si se quiere una democracia viva, si se aspira a una política compartida, se necesitan espacios y oportunidades que permitan debates abiertos, donde se construyan ideales y visiones también compartidas. Espacios en los que todos y cada uno puedan intervenir. Esas son las bases para poder hablar de ciudadanía, de inclusión social, de una nueva relación con la naturaleza. En definitiva, una sociedad en la que vale la pena vivir. (p. 6)

En concordancia con los agentes o actores educativos y la redefinición de su papel para vislumbrar un renovado sistema educativo, los organismos internacionales juegan un rol fundamental, debido a la influencia en las políticas educativas, dado que han venido promoviendo políticas estandarizadas para la educación no sólo en la región sino en el mundo. Según los autores Bonal y Verger (2011) el Banco Mundial lanzó el informe denominado La estrategia educativa 2020 para promover "El aprendizaje para todos", sin embargo, a criterio de estos autores: “...predomina un enfoque economicista tanto en el análisis como en las prescripciones relativas a la reforma educativa; el énfasis puesto en las políticas de mercado educativo y en el papel del sector privado; y la manera como el Banco trata la compleja relación entre pobreza y educación" (p. 917).

Esto significa que se pierde el enfoque integral de la política educativa, dado el sesgo economicista del Banco Mundial. Lo anterior invita a pensar en que el Estado requiere asumir su eficacia en virtud de las condiciones educativas que los organismos internacionales pudieran contribuir a propiciar, sin desconocer que son socios estratégicos, pero que el Estado deberá asumir la responsabilidad interna de los factores de atribución causal con respecto a la desigualdad educativa y social y a la vez defendiendo sus posiciones económicas a través del sistema político que abandera.

Otro de los actores que son considerados muy relevantes en la reconfiguración de un sistema educativo son los miembros de los gremios magisteriales. En el caso nacional, y conforme al criterio del sociólogo hondureño Posas (2019):

Las propuestas de lucha más globalizadoras han procedido de las centrales sindicales. Esto puede explicarse, en parte, porque aglutinan a trabajadores asalariados, a campesinos, a maestros y a otros sectores organizados, cuyos intereses tratan de 


\section{LA TRASCENDENCIA DE LAS POLÍTICAS EDUCATIVAS: UNA MEDIACIÓN PARA EL DESARROLLO HUMANO.}

reflejar en sus planteamientos. Sin embargo, sus preocupaciones han ido más allá de reflejar las demandas de los sectores que representan. Se han preocupado por democratizar el sistema político del país ofreciendo en este sentido una contribución que merece ser destacada. (p. 259)

Si bien, el movimiento magisterial es una fuerza válida, digna de ser reconocida ya que ha apostado a las reivindicaciones gremiales en toda la región, las cuales han sido importantes para resignificar la profesión docente, se percibe como limitada la capacidad de propuesta de sus proyectos educativos, que son capaces de romper con las desigualdades educativas y lograr el mejoramiento de los resultados.

Como núcleo organizado, se espera del gremio magisterial un planteamiento que advierta de un modelo educativo endógeno integrando una visión prospectiva, propositiva y de innovación curricular que genere una transformación en la sociedad. En el marco de los cambios que están reconfigurando los procesos educativos a nivel local y mundial, y siendo los movimientos magisteriales líderes de conocimiento en los temas educativos, se tiene la expectativa de que puedan armonizar criterios significativos con los otros actores, perfilando políticas integradoras y con una perspectiva de viabilidad a corto plazo.

Se infiere que una vez que el aparato estatal y la reforma de política educativa posicionen a los tomadores de decisión (autoridades educativas), considerados también actores educativos de incidencia, se impulse un proceso espiral hacia la reconfiguración del sistema educativo, es decir, con una postura hacia el acceso igualitario para todos/as $\mathrm{y}$, en segunda instancia, que reproduzcan un efecto catalizador, en donde los tomadores de esas decisiones estimen incluir proyectos que consideren las distintas voces y agendas, superando enfoques unidisciplinarios y de corte colonizador, esto según el autor mexicano Enrique Dussel quien postula que "...la reforma educativa signifique ser una visión de una historia distinta. Es decir, una descolonización mental” (2018, p. 46).

La resignificación de la educación tiene como reto asumir en sus contenidos programáticos el eje articulador de una formación ciudadana integral, conforme a lo que plantea Veramendí García (2012) autora que cita a D. Hillygus, autor de numerosos trabajos que vienen demostrando que es la educación ciudadana integral un factor que incrementa la participación política, la participación electoral, el comportamiento cívico, opiniones democráticas y el conocimiento político amparado 
en la ética reflexiva. Y supone que, si no se favorecen los proyectos formativos de estas, $\mathrm{u}$ otras habilidades pertinentes, mediados por un discurso activo de ciudadanía en la juventud, cabe la posibilidad de continuar propiciando condiciones de exclusión histórica en los ámbitos públicos.

Si no se hace énfasis en los valores democráticos en los sistemas educativos, entonces en el mejor de los casos los modelos educativos se encaminarán a educar a personas para que sigan asumiendo su rutina existencial. Según Apple W y Beane A. (2000) "Estas escuelas o bien son fútiles o bien subversivas. No tienen una razón legítima para existir" (p. 28). Y para superarlo se requiere un vuelco cualitativo del modelo educativo asumiendo la formación ciudadana como piedra angular y desde un enfoque sistémico.

Considerando los estudios sobre la formación ciudadana, los mismos podrían contribuir holísticamente a la resignificación del sistema educativo el cual está supeditado en buena parte por la buena gobernanza, rediseño de las políticas educativas y por un cuerpo de actores estratégicos y democráticos que asuman el reto de su transformación conforme a las necesidades educativas que apremian una atención de forma ineludible hacia los menos favorecidos.

\section{Horizonte de la educación para el desarrollo humano sostenible}

Actualmente existe una fuerte influencia de los mercados mundiales en el desarrollo de la sociedad, una tecnología de la comunicación y una producción invasiva; pero sobre todo en una gran crisis social de desconfianza. A esta crisis hay que sumarle los cambios acelerados de las nuevas generaciones. Generaciones de la inmediatez, como lo expone Baumann (2004) en su libro La ciudad sitiada, generaciones de riesgo global, marcada por los avances tecnológicos digitales se rigen por comunicaciones y convivencias mediadas por las nuevas tecnologías.

El mundo está cambiando, y si el entorno lo hace los actores también, ese cambio social involucra transformaciones internas y externas en la sociedad. El Estado garante de la justicia y promotor del bien común en un territorio determinado da paso a un mundo globalizado, a 'ciudadanos del mundo'. 


\section{LA TRASCENDENCIA DE LAS POLÍTICAS EDUCATIVAS: UNA MEDIACIÓN PARA EL DESARROLLO HUMANO.}

Las generaciones nacidas en los años 80's y 90's (millenials y centenialls) forman parte de un grupo poblacional educado en tecnología y en muchos casos mediante la tecnología, acostumbrado a satisfactores permanentes y efímeros que generan adicción, un claro ejemplo: las redes sociales. Estas generaciones crecen en un mundo de recompensas inmediatas, donde no hay que esperar, donde no hay necesidad de crear relaciones profundas y duraderas y mucho menos significativas.

Es en este contexto donde la educación, como mediadora de desarrollo humano, encuentra sus retos y sus desafíos. Es llegar a una generación que no crea arraigos con la familia, mucho menos con la sociedad y peor aún con el Estado; sin embargo, se quiere que puedan insertarse en un mundo donde nada les resulte extraño, un mundo digital donde tengan voz, donde opinen, donde cohabiten y donde ellos crean encajar. Se entiende que el rol fundamental de la educación es transformar a estas generaciones en esos ciudadanos del mundo, donde sea la precursora de las oportunidades sociales que menciona Amartya Sen y la que facilite la participación económica integrándose a un mercado laboral del siglo XXI y, al mismo tiempo, le garantice que sus derechos y libertades serán respetados (Sen, 1998).

Las crisis que azotan a la humanidad obligan al Estado a promover de manera prioritaria a la educación en todos los niveles como un bien común (con especial énfasis la educación terciaria) donde los valores de inclusión, solidaridad, desarrollo individual y colectivo inspiren al desarrollo sostenible de la nación, como apunta Gacel Ávila (2018), y que ya señalara la Declaración de la Conferencia Regional de la Educación Superior en ALC (CRES) de 2008 "La educación superior es un bien público social, un derecho humano y universal y un deber del Estado (...). Los Estados, las sociedades nacionales y las comunidades académicas deben ser quienes definan los principios básicos en los cuales se fundamenta la formación de los ciudadanos, velando por que ella sea pertinente y de calidad" (IESALC UNESCO, 2008).

Si bien este compromiso no es exclusivo del Estado es importante que lo lidere ante los diferentes actores que promueven el desarrollo. Es el Estado el que debe de manera permanente buscar una visión estratégica y holística del bien-estar humano; que genere sinergias entre los actores importantes con el fin de fomentar valores universales. No es que el Estado solamente priorice a la educación sobre otros sectores o que la vea como un derecho humano esencial y a base de desarrollo sostenible, es verla como una oportunidad de generación de conocimientos para la resolución de los problemas a los que se confrontan los ciudadanos. Es redireccionar 
la generación de esos conocimientos que, en los últimos 20 años, se ha enfocado, especialmente en América Latina, en áreas como la medicina, agricultura y ciencias biológicas, como apunta Ramírez (2018), y reenfocarlos en áreas de servicio que incidan en el crecimiento económico y a su vez que mejoren la calidad de vida de los ciudadanos.

Reconocer a la educación como derecho universal del hombre no es simplemente aumentar el acceso a la misma. Es reconocerla como el eje donde convergen las dimensiones culturales, sociales, económicas y políticas de la humanidad. Si bien hay claridad en que esta concepción que está en el centro del Programa de Desarrollo Sostenible 2030 (UNESCO, 2015) y que es fundamental para el logro de los 17 Objetivos del Desarrollo Sostenible (ODS 2030), no es claro poderla operacionalizar en los Planes de Nación de los países de la región. No sólo es el crecimiento de oportunidades de acceso a la educación de calidad lo que debe promoverse, sino la búsqueda de la democratización de la educación sobre la base de la colaboración entre sistemas y países. Es el fomento de una 'cultura educativa' donde se comparte y se colabora en la creación de nuevos conocimientos; donde los ciudadanos tengan acceso a marcos teóricos y conceptuales que les permitan de manera independiente y responsable el desarrollo de teorías para la solución a los problemas que se afrontan como sociedad. Como señala Guajardo (2018) es ver a los sistemas y a las instituciones como instancia social, asociados al desarrollo humano y al crecimiento de las sociedades; es promover un rol estratégico trasformador e integrador de la educación, en particular de la educación superior, como mediador del desarrollo humano sostenible de las naciones, especialmente en la región de América Latina y del Caribe.

La ventaja que presenta la generación de riesgo global es su resiliencia; su capacidad de adaptación que los hace superiores, apunta Ulrick Beck (2017). Esta resiliencia, que debe ser bastión de los sistemas educativos, debe aprovechar esta culturización digital obligada por crisis mundiales (COVID-19, por ejemplo) para adentrase en una transformación digital profunda y un aprovechamiento de la Inteligencia Artificial en pro de una democratización de la educación, como plantea Ramírez (2018), la información que se genera a través de las nuevas tecnologías de la información que se interconectan a través de internet es la nueva 'mina de oro' en la era digital. El crecimiento del internet puede ser una oportunidad de avance en la deseada democratización que se ha mencionado; y es que al democratizar el acceso al conocimiento y al saber se generan oportunidades para la construcción de la educación liberadora y contribuyen a la conquista de la ciudadanía, así como lo señala Guajardo (2018). La concepción de nuevos modelos que aprovechen las 


\section{LA TRASCENDENCIA DE LAS POLÍTICAS EDUCATIVAS: UNA MEDIACIÓN PARA EL DESARROLLO HUMANO.}

flexibilidades de la educación a distancia basadas en internet y la presencialidad, donde el espacio físico es para compartir y debatir ideas como decían Aristóteles, Platón y Sócrates es deseada y debe ser prioritaria en los años por venir para lograr una mejor cobertura.

La magnitud de oportunidades que brinda la digitalización es proporcional a sus retos. En el Plan de Acción de la CRES 2018 se habla de "Internet para TODOS", el reto no solo es de dotar de conectividad a los 4,100 millones de personas en el mundo que no poseen; sino también tratar otros problemas derivados de este ecosistema como son la ciberseguridad, la transparencia, la nube, la Inteligencia Artificial, la garantía de derechos fundamentales y la generación de valores especialmente los de ciudadanía y democracia.

\section{La educación y la ética: una propuesta para la participación ciudadana}

La participación ciudadana, un término que debe concebirse con responsabilidad, es la mediación para fortalecer las relaciones solidarias y empáticas en las sociedades del siglo XXI, sociedades mundializadas cuyo involucramiento es el potencial para lograr el desarrollo y bien-estar. Victoria Camps (2010) en su obra El declive de la ciudadanía. La construcción de una ética pública, en el capítulo 2-Democracias sin demos plantea la importancia de la ética en estas sociedades, afirmando que debe manifestarse desde aspectos como la solidaridad hasta la instauración de una forma de estado-gobierno, esto incluso al elegir, por ejemplo, a un representante gubernamental y en el uso de los caudales públicos (ya se ha instaurado la rendición de cuentas como requisito).

Vale la pena mencionar que según los principios de la ética se requiere que cada individuo viva la moralidad siendo consciente en quién se quiere ser, una moralidad de la vida manifestada en precisamente quién se es, una moralidad que englobe a la humanidad para considerar a quiénes y por quiénes somos, una moralidad de la igualdad que se medirá en la justicia y la equidad y, por último, en la moralidad de la comunidad cuando se tiene en cuenta el cómo debe proyectarse cada ser pensando en ese bien-estar de todos.

¿Por qué una propuesta de ética desde la educación para el desarrollo democrático en este siglo XXI?, aunque Zigmunt Bauman en Hernández Moreno (2003) habla de una modernidad líquida, donde hay vínculos frágiles y donde 
predomina una relación humana rápida, momentánea y superficial, se tiene que proponer la ética desde la educación para así lograr una participación constante y se acabe esa "...concepción individualista del sujeto democrático" (Camps, 2010, p. 18) porque sin participación no existe democracia. Es aquí donde hay que preguntar ¿por dónde comenzar?, pues a partir de una concepción donde la educación es lo esencial, para que sea desde ella que se enseñe la ética para la vida de forma constante y permanente, y así fortalecer a un ciudadano participativo y con ello consolidar la democracia. Por lo que Camps (2010) hace el llamado a una participación para que exista seguridad a lo que se pertenece, un compromiso al lugar de donde se procede y a quiénes se debe, ese sentido de identidad ciudadana imprescindible para enriquecer a la democracia. Un ciudadano capaz de intervenir en la toma de decisiones, de entender que su participación aportará a construir sociedades más justas.

Los valores, aunque arquetipos (como la libertad, el respeto y la tolerancia) aportarán a alcanzar objetivos comunes tanto políticos, como sociales y económicos que contribuirán a un desarrollo humano global, tan necesario en estos momentos de crisis. Por esto es por lo que se habla de la ética desde la educación, la que permitirá que haya confianza en las agendas gubernamentales, por ejemplo, así como servirá para hacer una elección de los líderes basados en todo un conjunto de valores reflejados por sus acciones destinados al bien común. Afirma Camps que debería instaurarse una “...educación para la ciudadanía” (p. 59), porque es desde allí que se le debe promover y desde edades tempranas. Para formar en la ética y así vincularse en la sociedad en una convivencia en armonía y en constante participación, como bien lo decía Aristóteles (citado por Camps, 2010), quien “....concibió la tarea moral como la de formar un carácter que hiciera de la persona alguien capaz de contribuir al mejoramiento de la polis" (p. 5) esta concepción es, sin lugar a dudas, una necesidad para crear democracia y con esto contribuir al desarrollo humano de las sociedades del mundo.

¿Cómo implementarlo? Por medio de una cultura política para la democratización mediada por la educación para así lograr “...el equilibrio entre libertad y responsabilidad en la vida ciudadana..." (Romero, 2014, p.15), este autor también menciona que se puede comenzar en “...liberar al Estado del control partidario... Controlar la corrupción... Integrar un equipo altamente profesional para gobernar, que incluya a los más capaces y honestos, más allá de su militancia partidaria" (p.16), es decir, desde una gobernanza desde la perspectiva de la ética. 


\section{LA TRASCENDENCIA DE LAS POLÍTICAS EDUCATIVAS: UNA MEDIACIÓN PARA EL DESARROLLO HUMANO.}

Se puede lograr participar desde la educación y Camps (2010) plantea que para que haya democracia "1) el ciudadano no es solo sujeto de derechos, sino también sujeto de deberes; 2) la ciudadanía consiste en ejercer la libertad responsablemente; 3) la responsabilidad es el momento afectivo de la formación de la voluntad moral" (p. 20). Esta misma autora propone que otra forma para atenuar la indiferencia a la participación es ser conscientes de la responsabilidad e implicación en la toma de decisiones para crear a un ser activo con ética ciudadana, porque "...la salud de una democracia no depende solo de la existencia de unas instituciones sino de la cooperación de una ciudadanía activa" (p. 18), afirmación que sirve para razonar la participación para la democracia, porque creará un entorno propicio para que la toma de decisiones esté en el control de los ciudadanos y que sean ellos (todos) los que hagan que los derechos regulen esas relaciones humanas, sin olvidar cumplir con los deberes.

\section{Articulación de las políticas públicas para la construcción de un sistema de gobernanza en educación}

De acuerdo con lo descrito en las secciones anteriores, los aspectos importantes para significar la definición de políticas públicas en general y, en particular, las políticas públicas para el sector educación en cuanto a que ningún Sistema Educativo, en cualquier país del mundo, podrá tener éxito o altos o medianos indicadores de logro si en principio el Estado no ha establecido en su quehacer gubernamental un modelo de gestión o, simplemente, una cultura institucional en cuanto a que el trabajo de las diferentes instituciones o sectores que atiende la problemática de la sociedad debe tener un alto grado de planificación, comunicación, objetivos comunes y con una visión prospectiva. Además, con un estilo de gestión donde la articulación de los programas y proyectos, así como la definición de las políticas públicas sean instrumentos y normativas creadas con la visión participativa y que se constituya en una red articulada de propósitos y acciones cuyo fin último, o resultados, logrados sea el de contribuir, subsanar, reducir la problemática social de los diferentes sectores que atiende el Estado para alcanzar propuestas y eventos de mejora continua para el logro del desarrollo humano en los diferentes espacios o comunidades que conforman la nación.

En esta parte se hace un alto para revisar de forma rápida el término de políticas públicas ligado al término bastante usado en la gestión pública y gobernanza, Vegas M.H. (2017) considera que “...la gestión pública es una estructura procedimental de acción gubernamental que lleva tareas de servicio a la sociedad a través de mecanismos legales y de ejecución política denominadas en muchas ocasiones como 
políticas públicas" (p. 2). Siendo el propósito de prestar servicio a los ciudadanos a fin de lograr una vida buena o con calidad.

Una buena gestión pública está muy relacionada con las acciones de la gobernanza. Parte de la construcción participativa, concertada, legitimada y socializada de las políticas públicas, las que serán articuladas en los diferentes estamentos o niveles de la planificación en los planes estratégicos, los planes operativos, a mediano y corto plazo. Destacamos aquí el concepto de la gobernanza. Según Vegas (2015) citado por Vegas Meléndez (2017) la gobernanza es “....una estrategia de acercamiento que permite incorporar diversos actores sociales e instituciones gubernamentales, o no, en la construcción de modelos autóctonos que permiten visualizar lo local desde la base social, partiendo del consenso/acuerdo; aspectos que validan una gestión vinculada a políticas públicas sustentables y de servicios públicos pensados en la ciudadanía” (p.164). Vegas M.H. (2017) citando a Vegas (2009), postula que una política pública “...representa un conjunto de acciones/actividades enmarcadas en normas y procedimientos regulatorios cuya orientación está dada a satisfacer necesidades colectivas promovidas por el Estado y por la presión social” (p. 6).

Pérez M.L.E. (2007) en su ensayo asegura que “...tradicionalmente las políticas públicas se diseñan y ejecutan sin tomar como referente las obligaciones constitucionales e internacionales del Estado en materia de derechos humanos"(p.143), por lo tanto "...los planes de desarrollo definen estrategias y programas sin valorar de manera suficiente su impacto sobre la realización de los derechos económicos, sociales y culturales" (p. 143), también plantea que “...las políticas educativas no siguen un enfoque de derechos humanos" (p. 143) limitando la "...efectividad del derecho a la educación" por lo que no se cumple con "...las obligaciones de respeto, protección y realización progresiva del mismo” (p. 143). En relación con cómo incluyen las políticas educativas en la planificación del Estado, Pérez M. L. E. (2007) menciona que en los planes de desarrollo se resalta el aspecto de la "...productividad y los rendimientos económicos" minimizando o ignorando “...la realización del derecho a la educación" (p. 148).

De acuerdo con Pérez M.L.E. (2007) “...un plan de desarrollo educativo que tenga como eje el derecho a la educación y la garantía de todos los derechos humanos en la educación debe empezar por reconocer en la planeación de la educación una política para realizar un derecho social" (p. 149). La complejidad de esta acción prevalecerá frente a "...la planeación de nueva infraestructura o de una política comercial” (p. 149). 


\section{LA TRASCENDENCIA DE LAS POLÍTICAS EDUCATIVAS: UNA MEDIACIÓN PARA EL DESARROLLO HUMANO.}

¿Entonces qué debe prevalecer en un Plan Nacional de Desarrollo? ¿Qué aspectos o principios serán fundamentales respecto a las políticas de educación? Las características que favorecen un buen Sistema Educativo Nacional se fundamentan en la definición de políticas educativas que respeten y signifiquen los principios del bien común, la educación como un derecho humano, la gratuidad de la educación como responsabilidad absoluta del Estado (M. L. Pérez, 2007, p. 150).

Tomaševski, K. (2001) citada por M. L. Pérez (2007), en su condición de Relatora Especial de las Naciones Unidas para el Derecho a la Educación asevera que:

...la efectividad de este derecho supone la realización simultánea de cuatro derechos y el cumplimiento de cuatro conjuntos de obligaciones por parte del Estado: el derecho a la disponibilidad de enseñanza y la obligación de asequibilidad, el derecho de acceso a la enseñanza y la obligación de accesibilidad, el derecho de permanencia en el sistema educativo y la obligación de adaptabilidad, y el derecho a una educación aceptable y la obligación de aceptabilidad. (p. 150)

Las políticas educativas nacionales tendrán como referente principal los conceptos y principios planteados por la UNESCO mediante las conferencias mundiales y regionales. Igualmente deberán estar articuladas al compromiso adquirido por el Estado a cumplir con el desarrollo y ejecución de la Agenda 2030 para el Desarrollo Sostenible aprobada en septiembre de 2015 por la Asamblea General de las Naciones, donde se detallan los 17 Objetivos del Desarrollo Sostenible en especial el Objetivo No. 4 donde se confirma que se debe "Garantizar una educación inclusiva, equitativa y de calidad y promover oportunidades de aprendizaje durante toda la vida para toda la consecución de una educación de calidad es la base para mejorar la vida de las personas y el desarrollo sostenible" (Informe ODS 2030, 2020, p. 5).

Estos 17 ODS serán articulados, entre sí, en el Plan Nacional de Desarrollo de País, así como en el Plan Sectorial de Educación Integral que sea representativo de los diferentes niveles de la educación prebásica (para la primera infancia) educación básica, media, educación técnica no universitaria, educación superior y, también, la educación no formal. "Los ODS también son una herramienta de planificación para los países, tanto a nivel nacional como local. Gracias a su visión a largo plazo, constituirán un apoyo para cada país en su senda hacia un desarrollo sostenido, inclusivo y en armonía con el medio ambiente, a través de políticas públicas e instrumentos de presupuesto, monitoreo y evaluación" (Informe ODS 2030, 2020, p. $5)$. 
Las políticas públicas para la sostenibilidad de los Sistemas Nacionales de Educación deberán llevar como propósitos fundamentales el uso eficiente y transparente de los recursos materiales e inmateriales asignados, la construcción de una educación inclusiva de la diversidad, el acceso de la mayoría o a los sectores que requieran de una educación formal o educación no formal, técnica no universitaria, educación técnica universitaria, también universitaria en licenciaturas, especialidades, maestrías, doctorados y posdoctorados.

Igualmente, una política pública coherente, pertinente y articulada con las otras dimensiones o sectores productivos, cuyas acciones educativas incidan en las dimensiones de la diversidad ambiental, ecológica, económica, salud y social (cultura, arte, deportes). Estas políticas públicas articuladas podrán ejercerse con lógica e interés ciudadano, y de autoridades gubernamentales que son conscientes y poseen un compromiso social y de nación para lograr resultados propuestos en los Planes Estratégicos del Estado-Gobierno, Planes Operativos Institucionales, Plan Estratégico del Sector Educativo (PESE), Plan Estratégico de la Educación Superior, de Comunidades y de Sectores (DES, Informe I, 2018).

\section{La educación: planificación en prospectiva desde las universidades}

El Sistema Educativo en sus diferentes niveles y en especial el Sistema de Nivel Superior siguen siendo el referente y el espacio público para el diálogo, la formulación de política pública, el establecimiento de estrategias de gobernanza (Dirección de Educación Superior-DES 1988, Ley de Educación Superior-LES y sus reglamentos 1989).

El nivel de Educación Superior representado a través de las instituciones de educación superior de las universidades hondureñas es el espacio público y la dimensión que convierte a la educación con calidad, inclusión, equidad, pertinencia, es la alternativa que hará que los hondureños logren un desarrollo humano sostenible, para lograr solventar la superación de la inmediatez (Normas Académicas del Nivel de Educación Superior de Honduras-1994, A. Serrano y H. Stein, 2021).

Las instituciones de educación superior dentro de sus visiones y misiones están orientados a desarrollar un compromiso social, una educación de calidad, ofertar la formación con pertinencia y gozar de una autonomía institucional académica y o financiera (Normas Académicas del Nivel de Educación Superior de Honduras, 1992). 


\section{LA TRASCENDENCIA DE LAS POLÍTICAS EDUCATIVAS: UNA MEDIACIÓN PARA EL DESARROLLO HUMANO.}

Las instituciones de educación superior tienen el propósito de la formación profesional por medio de sus tres funciones esenciales la docencia, la investigación y la vinculación desarrollando la ciencia y la tecnología, además, tiene la obligación de construir y generar cultura, desarrollar las artes, las humanidades, la ética, la identidad y la ciudadanía (Ley de Educación Superior de Honduras-LES y sus reglamentos 1989; V. G. Alfaro, 2021).

La planificación en prospectiva es una condición fundamental para afrontar las realidades y los retos existentes dentro del Sistema Educativo Nacional y en el espacio sociedad, ciudad y comunidad local (Calderón S. R., 2021). En relación con esto la UNESCO ha iniciado un proyecto denominado Los Futuros de la Educación Superior hasta el 2050. El 25 de mayo de 2021 ha presentado el Informe Pensar más allá de los límites, perspectivas sobre los futuros de la educación superior hasta 2050, estos constituyen los primeros avances en la etapa inicial de consultas a expertos. A continuación, el resumen de algunos aspectos importantes de acuerdo con el informe: Los mensajes claves se han fusionado en cuatro amplias declaraciones sobre el futuro de la educación superior. En esta visión compuesta, la educación superior asume una responsabilidad activa por nuestra humanidad común:

- Abre y desarrolla el potencial de todos los seres humanos.

- Afronta los riesgos y tiende puentes entre el tiempo, las personas y los lugares.

- Defiende el conocimiento y las formas de saber, en tanto que es un bien público mundial. Promueve el bienestar y la sustentabilidad.

- Se orienta hacia la justicia, la solidaridad y los derechos humanos.

- Respalda un proyecto de vida que fortalece a las personas, sus familias, las comunidades y la humanidad.

- Actúa y se organiza de manera ética, sostenible y responsable. Se nutre de la diversidad intercultural y epistémica.

- Preserva las identidades y las culturas, ya sean colectivas, institucionales o personales.

- Crea espacios para la reflexión y el diálogo.

- Realiza comparaciones de buena fe, sin imponer ni presuponer homogeneidad. Defiende y crea interconexiones a múltiples niveles.

- Fragua colaboraciones entre personas, grupos y comunidades locales y globales.

- Mantiene los vínculos entre las IES, los niveles de educación y el aprendizaje formal e informal. 20

- Relaciona a los seres humanos con los seres no humanos, la tierra y el universo (UNESCO-IESALC, 2021, pp. 49-50). 
De acuerdo con este resumen aportado por los expertos, consultados con la UNESCO, se confronta con una realidad presente en donde prevalecen las inequidades de la humanidad contra la humanidad, aquellas que se deben comenzar a eliminar; la educación es el punto focal, es la acción que por excelencia ha cambiado la vida de muchos ciudadanos del mundo, pero esto no debe ser acosta de la carencia o la falta de asequibilidad, accesibilidad, de adaptabilidad y de aceptabilidad de las mayoría de la población mundial a los procesos educativos; así también el resumen desafía a los Sistemas de Educación Nacional y en especial al Nivel de Educación Superior a proporcionar ideas, conceptos y propuestas innovadoras de acción, en los diferentes campos del conocimiento, al Estado, gobierno, empresas, comunidades a la sociedad en general; son las universidades públicas o privadas las llamadas a actuar en colaboración aportando para la gestión pública y la ejecución de la política pública, generando ciencia, investigaciones, debate y soluciones, todo ello para el bien común, para que la educación se constituya en uno de los factores más fuertes para el logro del desarrollo humano sostenible.

Debe existir un replanteamiento del enfoque educativo que lleve a alcanzar la transformación de la educación con una perspectiva crítica y epistemológicamente emancipadora frente a la hegemonía de modelos educativos que, aún en el siglo XXI, se perciben bizantinos y perpetuadores de la segregación.

La transformación digital educativa pasa por un consenso y alianza entre los diferentes actores: Estado, empresas e Instituciones de Educación. Alianza que es fundamental en estos tiempos difíciles donde el futuro ya llegó, y que exige un compromiso inmediato para el desarrollo sostenible. Es una visión sistémica y, a largo plazo, que se debe priorizar en estos momentos de incertidumbre. El desarrollo de una 'cultura educativa' no es solamente una acción, es una estrategia que debe priorizarse. Se deben ampliar los modelos, adaptarlos a los nuevos actores y a las nuevas generaciones que son más escolarizadas, pero con peores perspectivas que las anteriores, lo que provoca una interacción o cohabitación conflictiva y desafiante.

Por consiguiente, la reconfiguración del sistema educativo estaría sujeta a un nuevo planteamiento de las políticas educativas, lo cual equivaldría a una auténtica apuesta, encaminada a una ruptura estructural en la continuidad programática de las políticas educativas previamente establecidas, que a la vez apunten a determinadas acciones estratégicas, tanto para generar indicadores educativos satisfactorios como al desarrollo humano en los países. 


\section{LA TRASCENDENCIA DE LAS POLÍTICAS EDUCATIVAS: UNA MEDIACIÓN PARA EL \\ DESARROLLO HUMANO.}

\section{BIBLIOGRAFIA}

Alianza Global. (2014). Hacia una cooperación al desarrollo más eficaz: informe de avances. Capítulo 4-Transparencia y rendición de cuentas para los resultados del desarrollo. https://www.oecd-ilibrary.org/docserver/9789264209718-8-es.pdf?expire $\mathrm{s}=1624896046 \& \mathrm{id}=\mathrm{id} \&$ accname $=$ guest $\&$ checksum $=\mathrm{E} 51 \mathrm{D} 7 \mathrm{ADD} 81 \mathrm{C} 5 \mathrm{C} 1 \mathrm{~B} 46 \mathrm{~F} 1 \mathrm{~B} 9$ 17C939A83C1

Alfaro, V. G.,(10 mayo-30 junio 2021). Papel de la Educación Superior en la promoción del Desarrollo Humano Sostenible. Eje Educación y Desarrollo. Presentación, Foro Debate Contemporáneo sobre las Ciencias del Desarrollo Humano. Doctorado en Ciencias del Desarrollo Humano-UNAH.

Apple W, M., y Beane A, J. (2000). Escuelas Democráticas. En ¿qué es una escuela democrática? (p. 24).

Arnaiz Arnaiz, Natxo. (2010). Siglo XXI: ¿el siglo de la participación? Agencia para el voluntariado y la participación social de Bizkaia.

Banco Interamericano de Desarrollo. (2020). La crisis de la desigualdad, América Latina y el Caribe en la entrecrucijada.

Bauman, Z. (2011). Daños colaterales, desigualdades sociales en la era global. Fondo de Cultura Económica (FCE).

Bauman, Z. (2004). La sociedad sitiada. Fondo de Cultura Económica

Bechara Llanos, Abraham Zamir. (2015). Aproximación teórica al concepto de estado: Distinciones en torno a Heller, Jellinek y Carré De Malberg. Revista Jurídica Mario Alario Filippo, VII (14), p. 72-84.

Beck, U. (2000). Retorno a la teoría de la sociedad del riesgo. Boletín de la Asociación de Geógrafos Españoles.

BECK, Ulrich. (2017). Las generaciones del riesgo global: unidas en la decadencia, en la metamorfosis del mundo. Paidós.

Beck, Ulrich. (2017). La metamorfosis del mundo. Comunidades de riesgo cosmopolitas: De las naciones unidas a las ciudades unidas. Pp. 149-178.

Bonal, X., y Verger, A. (2011). La estrategia educativa 2020 o las limitaciones del 
Banco Mundial para promover "El aprendizaje para todos”. Scielo, Vol. 32, No. 117, pp. 911-932.

Cáceres Valladares, D. F. (2018). Calidad y política educativa para el desarrollo humano. (Tesis Doctoral). Universidad Nacional Autónoma de Honduras.

Calderón, S. R. (10 mayo-30 junio, 2021). Desarrollo, potencialidades y aportes de la Educación Superior en América Latina y el Caribe. Perspectiva del IESALC/ UNESCO. Presentación, Foro Debate Contemporáneo sobre las Ciencias del Desarrollo Humano. Doctorado en Ciencias del Desarrollo Humano. UNAH.

Camps, Victoria. (2010). El declive de la ciudadanía. La construcción de una ética pública.

Consejo de Educación Superior-CES. (1992). Normas Académicas del Nivel de Educación Superior. La Gaceta No. 26911.

Dacombe, R., y Pharvin, P. (2021). Participatory democracy in an age of inequality. Representation, journal of representative democracy. P. 4.

Dirección de Educación Superior. (2018). La Educación Superior en Honduras y sus líneas estratégicas de desarrollo. Informe de investigación.

Dussel, E. (2018). La transformación de la educación hacia la descolonización de la pedagogía. https://www.youtube.com/watch?v=sWg94cBYDrM

Fontaine, G. (2015). El análisis de políticas públicas. Conceptos, teorías y métodos. Anthropos.

Gamallo, Gustavo. (2006). Participación ciudadana en América Latina: La participación como un componente del concepto de ciudadanía. Perspectiva.

Guajardo, P. H., Mato, D., Grimaldo, H., Gacel-Ávila, J., Lemaitre, M. J., Guarga, R., y Ramírez, R. (2018). Tendencias de la educación superior en América Latina y El Caribe.

Hernández Moreno, Jazmín. (2016). La modernidad líquida. Política y cultura No. 45. http://www.scielo.org.mx/scielo.php?script=sci_arttext\&pid $=$ S0188-77422016000100279

IDEA Internacional. (2017). El estado de la democracia en el mundo. Examen de 


\section{LA TRASCENDENCIA DE LAS POLÍTICAS EDUCATIVAS: UNA MEDIACIÓN PARA EL DESARROLLO HUMANO.}

la resiliencia democrática. https://www.idea.int/gsod-2017/files/IDEA-GSOD-2017OVERVIEW-ES.pdf

IESALC-UNESCO. (2008). Declaración y Plan de Acción de la Conferencia Regional de Educación Superior en América Latina y el Caribe. www.unesco.org.ve. www. unesco.org.ve/documents/DeclaracionCartagenaCres.pdf

Lorente Rodríguez, M. (2019). Problemas y limitaciones de la educación en América Latina. Un estudio comparado. Foro de Educación, 27(17), 229-251. doi:http:// dx.doi.org/10.14516/fde.645

Miranda, Francisco Javier. (2008). La configuración de la identidad ciudadana en un contexto multicultural. Praxis Filosófica No. 27. https://www.redalyc.org/ pdf/2090/209014644008.pdf

Naciones Unidas. (2021). Informe sobre los progresos en el cumplimiento de los ODS-Desarrollo Sostenible. www.un.org/sustainabledevelopment/es

Pérez Murcia. (2007). La exigibilidad del derecho a la educación a partir del diseño y la ejecución de las políticas públicas educativas. Revista Estudios Socio-Jurídico, 9 (Número especial). Pp. 142-165.

Reimers, F. (2000). Educación, desigualdad y opciones de política en América Latina en el siglo XXI. Revista Latinoamericana de Estudios Educativos, pp. 11-42.

Serrano L. A. y Stein H. A. (10 mayo-30 junio, 2021). Desarrollo Humano Sostenible. Foro Debate Contemporáneo sobre las Ciencias del Desarrollo Humano. Doctorado en Ciencias del Desarrollo Humano. UNAH.

Subirats, J. (2011). Otra sociedad ¿otra política? de «no nos representan» a la democracia de lo común. Icaria editorial.

Rojas, A. (2020). Desafíos en calidad y cobertura de la educación pública de Honduras 2014-2018. Revista Economía y Administración, 11(2), 9-23. doi:10.5377/ eya.v11i2.10517

Romero Ramón. (2014). Honduras: la difícil opción democrática. http://biblioteca. clacso.edu.ar/clacso/becas/20140905114750/1234.pdf

Transparencia Internacional. (2020). Honduras ocupa el segundo lugar en corrupción en Centroamérica. El País. https://www.elpais.hn/2020/01/23/honduras-ocupa-el- 
segundo-lugar-de-corrupcion-en-centroamerica-segun-transparencia-internacional/ Torres Bugdud, Arturo, Álvarez Aguilar, Nivia y Del Roble Obando Rodríguez María. (2013). La educación para una ciudadanía democrática en las instituciones educativas: Su abordaje socio pedagógico. Revista Electrónica Educare Vol. 17, No. 3, [151-172]. http://www.una.ac.cr/educare

UNAH. (2005). Plan General para la Reforma Integral de la UNAH.

UNAH. (1989). Ley de Educación Superior. Decreto del Congreso Nacional No.14289. La Gaceta No. 25961.

UN-CEPAL. (2018). Panorama de la Gestión Pública en América Latina y el Caribe. Un gobierno abierto centrado en el ciudadano. https://www.cepal.org/sites/default/ files/publication/files/42396/S1701222_es.pdf

UNESCO. (2018). Conferencia Regional de Educación Superior de América Latina y el Caribe. Integración y conocimiento.

UNESCO. (2020). Informe de seguimiento de la educación en el mundo 2020. América Latina y El Caribe, Inclusión y educación: Todos y todas sin excepción.

UNESCO/IESALC. (2021). Pensar más allá de los límites. Perspectivas sobre los futuros de la educación superior hasta 2050. http://creativecommons.org/licences/bysa/3.0/igo/Repositorio UNESCO de acceso abierto. www.unesco.org/open-access/ terms-use-ccbysa-sp

Vargas, C. (2019). Leaving no one behind: bringing equity and inclusion back into education. Resisting Neoliberalism in Education: local, national and transnational perspectives, L. Tett y M. Hamilton (Eds.), Bristol, Policy Press.

Vásquez Daniel. (2020). Honduras en el abismo. Nueva Sociedad, No. 287. https:// nuso.org/articulo/honduras-en-el-abismo/

Vegas, Meléndez, H. (2017). El Estado en América Latina: un análisis desde las Políticas Publicas y gobernanza: Articulación para una gestión pública local autónomo. Edición electrónica http://journals.openedition.org/polis/12661

Veramendí García, M. (2012). Educación y ciudadanía, análisis de la relación entre trayectoria educativa, literacidad, ethos y praxis política de una muestra de adultos de un distrito de Los Andes peruanos. Arteta. 


\section{CÍRCULO TEMÁTICO: EDUCACIÓN Y DESARROLLO}

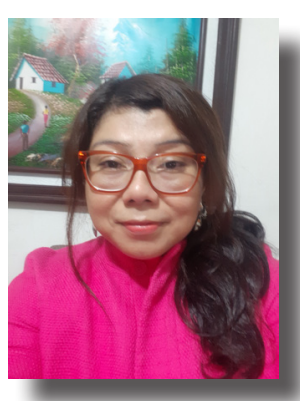

Lorena Suazo

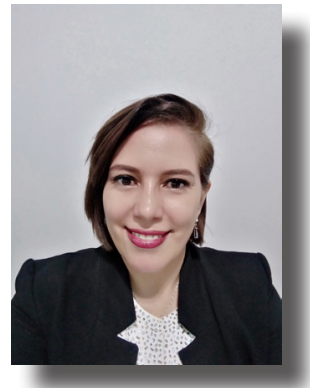

Marlenne Ordóñez

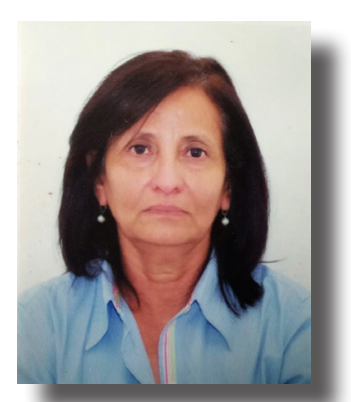

Rosa Elia Sabillón

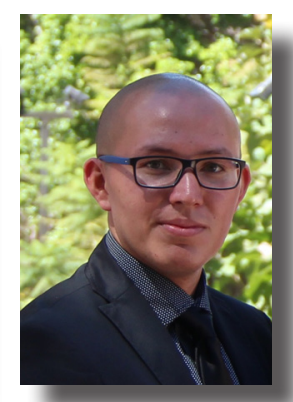

Orlando Martínez

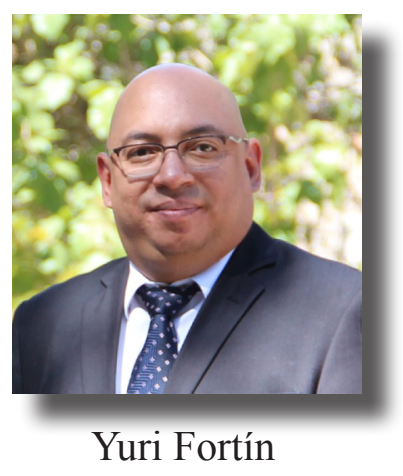

